\title{
Monitoring Endangered Species Populations: Gene Dispersal Can Have Pronounced Effects on the Relationship between Census Size and Genetic Diversity
}

\author{
Steven H. Rogstad $^{1 *}$, Stephan Pelikan ${ }^{2}$ \\ ${ }^{1}$ Department of Biological Sciences, University of Cincinnati, Cincinnati, USA; ${ }^{2}$ Department of Mathematical Sciences, University \\ of Cincinnati, Cincinnati, USA. \\ Email: ${ }^{*}$ steven.rogstad@uc.edu
}

Received June $21^{\text {st }}, 2013$; revised July $22^{\text {nd }}, 2013$; accepted August $15^{\text {th }}, 2013$

Copyright (C) 2013 Steven H. Rogstad, Stephan Pelikan. This is an open access article distributed under the Creative Commons Attribution License, which permits unrestricted use, distribution, and reproduction in any medium, provided the original work is properly cited.

\begin{abstract}
Anthropogenic activities are increasing habitat fragmentation, as well as the number of threatened and endangered species. Thus, isolated fragments with natural remnant stands, or in situ or ex situ endangered species rescue populations, are on the rise. The most common method for assessing the "conservation health" of such populations is to determine or estimate the census size. However, while it is known that the census size of a population does not always correlate with its genetic diversity, methods for modeling how different factors can drive variation in the relationship of census size to genetic diversity in plant populations are needed. Here we use the computer program NEWGARDEN to investigate how the relationship of stand size versus genetic diversity (measured as both the percent of the founding alleles retained and $\mathrm{F}_{\mathrm{IT}}$ ) can be extremely variable depending on founder number, founder density, and gene dispersal distances. Populations of endangered species that appear to have the same conservation health in terms of similar population numbers may differ greatly in their conservation health as indicated by the genetic diversity they retain. NEWGARDEN can be used to explore how different founding and intra- or interspecific life history characteristics can affect genetic diversity relative to census size. If proper historical data exist, NEWGARDEN can also be used to estimate the percent of founding genetic diversity remaining in a given stand.
\end{abstract}

Keywords: Biodiversity; Fragmented Populations; Plant Population Modeling; Population Genetics; Restoration Management; Species Conservation

\section{Introduction}

The protection of endangered plants includes maintaining viable populations while preserving their genetic diversity. Endangered species commonly exist in small, isolated, and/or low density populations. In situ and ex situ restoration or rescue populations will also often share these characteristics. Such small populations are especially susceptible to loss of genetic diversity via random genetic drift and inbreeding. The latter two processes may drive reduced fitness for individuals and populations due to inbreeding depression and genostasis (decline of genetic variability resulting in a loss of potential evolutionary responses) [1]. Therefore, the monitoring and maintenance of genetic diversity in populations of endangered species are highly recommended [2].

\footnotetext{
"Corresponding author.
}

One suggested guideline rule for the minimum size of populations is the so-called " $50 / 500$ " rule attributed to Franklin [3] and Soulé [4], according to which a population must have an effective size of 50 breeding individuals to prevent inbreeding and 500 individuals to prevent loss of genetic variation via drift. It is well known that since these numbers refer to ideal effective population sizes (i.e., $\mathrm{N}_{\mathrm{e}}$ ), and most populations do not perform as ideal populations, $\mathrm{N}_{\mathrm{c}}$ (the actual census size of a population) is usually greater than $\mathrm{N}_{\mathrm{e}}$ [2]. Reviewing data from studies on over 100 animal and plant species, Frankham [5] estimated that, on average, $\mathrm{N}_{\mathrm{c}}$ is approximately ten times greater than $\mathrm{N}_{\mathrm{e}}$ in wild populations. Thus, the $\mathrm{N}_{\mathrm{e}}$ $50 / 500$ rule becomes the $N_{c} 500 / 5000$ rule. But other authors have argued that this is both an overestimate [6] or an underestimate (in some cases by orders of magnitude [7]) for the size of $N_{c}$ relative to $N_{e}$. For example, it has 
been proposed that the $\mathrm{N}_{\mathrm{e}} 50 / 500$ numbers are too small, and that populations with $\mathrm{N}_{\mathrm{e}}$ sizes approaching at least 1000 to 5000 are needed in the long term to prevent "mutational meltdown" stemming from the accumulation of deleterious mutations in smaller populations (e.g., [810]).

However, many factors contribute to the realized $\mathrm{N}_{\mathrm{e}}$ values and rates of drift for populations through time, including variable mating systems, demographics, and gene dispersal distances. Thus, estimating the $\mathrm{N}_{\mathrm{e}}$, and more importantly for endangered plant conservation considerations, the rates of loss of genetic diversity in natural populations, are difficult. Census size may be deceptive as to the genetic diversity retained by a population, but, short of extensive genetic surveys, how can a manager model what has occurred in the population to gain an estimate of current diversity retained?

Adding to the complexity of these considerations, for many endangered species, obtaining and successfully establishing 500 or 5000 , or even 50 , genetically distinct viable propagules can be a challenge, and restoration methodologies that preserve genetic diversity in such small populations are needed. But conducting sufficiently replicated field experiments to determine general guidelines on how best to manipulate newly founded populations to secure stand maintenance and genetic variation going forward is cost-prohibitive and not feasible, especially given the array of life-history characteristics distributed across the wide range of endangered plant species.

To statistically explore the above issues, we have produced the computer program NEWGARDEN [11,12], which generates virtual plant populations that develop through generations as conditioned by input parameters concerning available habitat size and shape, the size and geometric placement of the founding population, and the life history characteristics of the study species. Future generations develop via matings and offspring production processes emerging from the initial input parameters. Throughout a user-specified number of generations (bouts of mating), the program provides statistically analyzable output for the total population (including all age classes) concerning population growth and genetic diversity (see below). In the comparative trials presented here, we use NEWGARDEN to explore, for an annual plant (lacking a seed bank), how varying founder number and density, combined with varying gene dispersal distances, can affect population growth and genetic diversity of populations. Our results demonstrate that plant populations initiated with the same number of founders can attain identical subsequent population sizes that retain genetic diversity to widely varying degrees due to differences in gene dispersal. Replicated plant preservation projects may appear to have similar population sizes, but such stands may have very different levels of genetic variation.

\section{Materials and Methods}

All trials were analyzed using NEWGARDEN version 2.2 (4apr2012). One set of user-defined input parameters is called a "trial". For each trial, the user specifies the number of "runs", that is, replicate re-runs of those particular trial input conditions. With output statistics being produced and stored for each individual run, the ultimate summary output (which is what we report below) is provided in the form of mean values across runs with standard deviation for each output value. In all of the trials analyzed here, such summary output values (see below) represent the mean of 100 replicate runs.

Except as noted below, the following input parameter conditions were held constant across all trials (parameters are discussed in order that they appear in input files). Each individual was analyzed at 20 loci, each locus having 100 alleles at equal frequencies in the source population (frequency $=0.01$ ). Each individual was cosexual. The expected offspring production per generation per adult was 1.017, distributed among eligible individuals according to the Poisson distribution. All individuals could produce pollen. The species is an annual. The isolated preserve is 2001 by 2001 grid points in size (carrying capacity, or $\mathrm{K}=44,004,001$ grid points). Individuals can exist only on grid points, which are meant to represent the average minimum distance between individuals when the local population is at $\mathrm{K}$. If the distance between points here is $1 \mathrm{~m}$, then the preserve is approximately $2 \mathrm{~km}$ on a side. Populations developed over 34 bouts of mating (generations) unless noted. The source population from which alleles were drawn for the founders was at Hardy-Weinberg equilibrium.

Other conditions varied among trials. Although founders were always scattered randomly throughout the preserve, comparative trials were initiated with either 1000 (high density) or 300 (low density) founders. For each density condition, 14 trials were run which differed either in the maximum distances from which pollen in a given mating could arrive, or to which offspring were dispersed. These differences in gene dispersal will be described where results of comparative trials are discussed below. To explain an example, in one trial, maximum offspring dispersal distance might be five grid points with a pollen dispersal maximum of 250 grid points. This means that in a given randomly selected mating, offspring can be dispersed to any point that is within five grid points in the $\mathrm{x}$ or $\mathrm{y}$ direction (each axis distance randomly selected separately), and pollen will come from a randomly selected pollen source that is within $250 \mathrm{x}$ and $\mathrm{y}$ grid points of the selected pistillate individual. Again, $\mathrm{x}$ and $\mathrm{y}$ dis- 
tances are randomly selected from within that range. In other words, centered on the selected pistillate plant in a mating, offspring can be randomly dispersed to one of the nearest 121 grid points, and pollen can come from a pollen-producing plant within the nearest 251,001 grid points. Under these conditions, if an offspring is dispersed off the preserve, it "dies" (is removed from further analyses). If it is dispersed to a point to which other offspring are also dispersed, one is chosen to establish there at random, the others dying. If no pollen donor can be found within the specified distance range for a particular mating, the mating fails but is counted as one of the mating events for a generation.

Output includes mean values for total population size, number of founding alleles remaining, and $\mathrm{F}_{\text {IT }}$ [13], a measure of population inbreeding and differentiation as explained below. Data points discussed as being different differ significantly in mean value at the $p$-value $\leq 0.05$ criterion. More details regarding the NEWGARDEN program are provided elsewhere $[11,12]$.

\section{Results}

In our first example (Figure 1), populations were initiated with either 1000 founders (letters in upper case) or 300 founders (lower case letters). Since trials were initiated with different numbers of founders, change in population size is expressed as the percent of the original founders remaining through sequential bouts of mating.

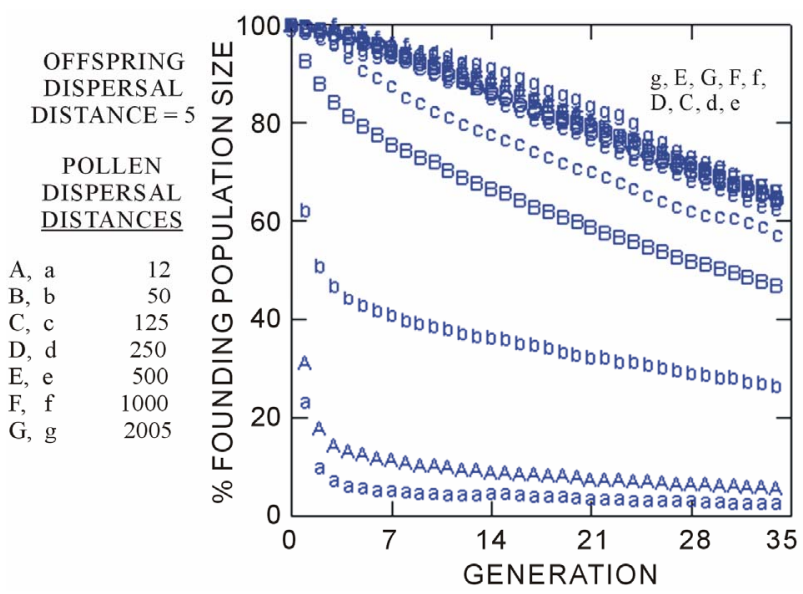

Figure 1. Continuing population size as a percent of the original number of founders through generations for NEWGARDEN populations initiated with 1000 founders (letters in upper case) or 300 founders (lower case) scattered at random throughout the preserve. In these trials, maximum offspring dispersal is always 5 grid points, while maximum pollen dispersal distances (in grid points) vary as indicated in the key to the left of the graph. The letters at the upper right of the graph denote, in descending order, the trials clustered at generation 34 to the upper right of the graph. See text for more details.
In this example, trials have identical conditions, including a constant maximum offspring dispersal distance of 5 grid units (distributed to a point chosen at random within the nearest 121 grid units), while trials with the same number of founders vary only in that the maximum distance across which pollen is dispersed from a randomly chosen eligible staminate individual to a given pistillate off spring-producing plant differs as indicated in the legend to the left of the graph. While the exact same reproductive value has been input for all of these trials $(r=$ 1.017), and all populations are declining in size, they decline at different rates depending on the maximum distance from which pollen can arrive in a given mating. When pollen dispersal is relatively short as in trials a, A, $\mathrm{b}, \mathrm{B}$, and to some degree, $\mathrm{c}$, the populations decline noticeably more rapidly than for the other trials (grouped as listed in descending order at the upper right of the graph). In other words, this graph shows that if a fragmented, isolated population initiated with 1000 or 300 scattered individuals experiences relatively limited offspring and pollen dispersal distances, then populations will decline more rapidly.

Figure 2 shows that these same trial populations are more variable as to the degree of genetic diversity loss

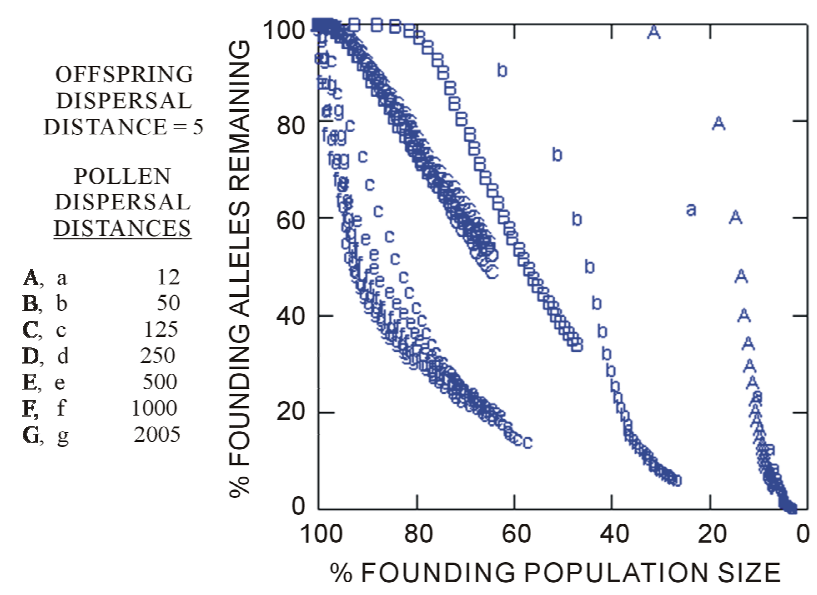

Figure 2. Percent of the founding alleles remaining relative to the size of the continuing population as a percentage of the original number of founders for the same trials for which population development data are depicted in Figure 1. These NEWGARDEN populations were initiated with 1000 founders (letters in upper case) or $\mathbf{3 0 0}$ founders (lower case) scattered at random throughout the preserve. In these trials, maximum offspring dispersal is always 5 grid points, while maximum pollen dispersal distances (in grid points) vary as indicated in the key to the left of the graph. When populations attain $80 \%$ of their founding size, the percent of the founding alleles remaining ranges from approximately $100 \%$ (e.g. trials A, a, b, and B) to approximately $30 \%$ (e.g., trial g). Percent of the founding alleles remaining depicted at $80 \%$ founding population size, in vertically descending order, are: A,a,b,B,F,G,E,D,C,c,e,f,d,g. Values interpolated when necessary. 
when populations have lost equal percentages of individuals compared to the original founders. For example, consider all of the trial populations when they have declined to $80 \%$ of their founding size. Trials A, a, b, and B still retain approximately $100 \%$ of the founding alleles when their population size is $80 \%$ that of the founders. For the remaining trials at $80 \%$ of their initial founding size, populations initiated with 1000 founders retain from approximately $73 \%$ (trials $\mathrm{C}, \mathrm{D}$, and $\mathrm{E}$ ) to $77 \%$ (trials $\mathrm{F}$ and $\mathrm{G}$ ), and when founded with 300 individuals, allele retention is approximately $31.3 \%$ (trials $\mathrm{d}$ and $\mathrm{g}$ ) to $41.7 \%$ (trial c). These trials also differ markedly in F values (Figure 3). Consider again trial results when populations have declined to $80 \%$ of their founding size. $\mathrm{F}$ values range from 0.07 (interpolated for trial A), suggesting moderate inbreeding and genetic differentiation within the population, to 0.54 (trial c) indicating that a very great degree of inbreeding and genetic differentiation have occurred in the population [13]. All of these trials have limited offspring dispersal distance (5 grid points). But higher levels of $\mathrm{F}$ are achieved when trials have maximum pollen dispersal distances ranging from 12 grid points (maximum $\mathrm{F}$ value for trial $\mathrm{A}=0.82$ ) to 500 grid points (trial e). Maximum pollen dispesal distances to longer distances reduces $\mathrm{F}$ values to $<0.14$ (moderate

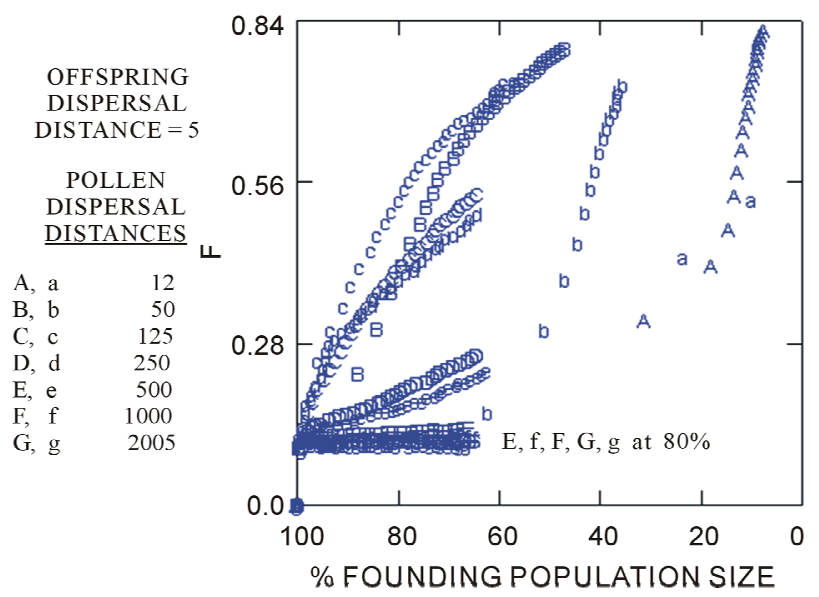

Figure 3. F values relative to the size of the continuing population as a percentage of the original number of founders. These are the same trials for which population development data are depicted in Figure 1. These NEWGARDEN populations were initiated with $\mathbf{1 0 0 0}$ founders (letters in upper case) or 300 founders (lower case) scattered at random throughout the preserve. In these trials, maximum offspring dispersal is always 5 grid points, while maximum pollen dispersal distances (in grid points) vary as indicated in the key to the left of the graph. When populations attain $80 \%$ of their founding size, $F$ values range from approximately 0.5 (trials c) to approximately 0.07 (trial A). F values depicted at $80 \%$ founding population size, in vertically descending order, are: c,B,C,d,D,e,E,f,F,G,g, with interpolated values of 0.095 for $a, 0.08$ for $b$, and 0.07 for $A$. inbreeding and subdivision).

In another set of trials, the maximum distance from which pollen could arrive for a particular mating was fixed at 2005 grid points in all trials, with trials varying only as to the maximum grid point distance that offspring could be dispersed (Figures 4 and 5). While all of the trials considered previously were declining through generations (Figure 1), in this set of trials, some populations grew while others declined (Figure 4), even though $r$ was set constant at 1.017 as before. As in the previous trials, the percentage of founding alleles remaining varies considerably across trials relative to the percent of the founding population remaining through generations (Figure 5). For example, populations that have declined to $50 \%$ of the founding size may have $17 \%$ (trial d) to $100 \%$ (trials $\mathrm{F}$ and $\mathrm{G}$ ) of the founding alleles remaining. To examine these data from a different perspective to include all trials, when the percentage of founding alleles remaining in all trial populations is $80 \%$, population size relative to the initial number of founders ranges from approximately $125 \%$ (trial A) to $14 \%$ (trial G). In other words, when populations retain equal amounts of founding alleles, they can vary considerably in their size relative to the founding population due to differences in offspring dispersal. $\mathrm{F}$ values for this second set of trials with varying offspring dispersal ranged from approximately 0.09 (trials $F$ and f) to 0.135 (trials $G$ and g), suggesting moderate levels of inbreeding and genetic differentiation were occurring in all of these populations (data not

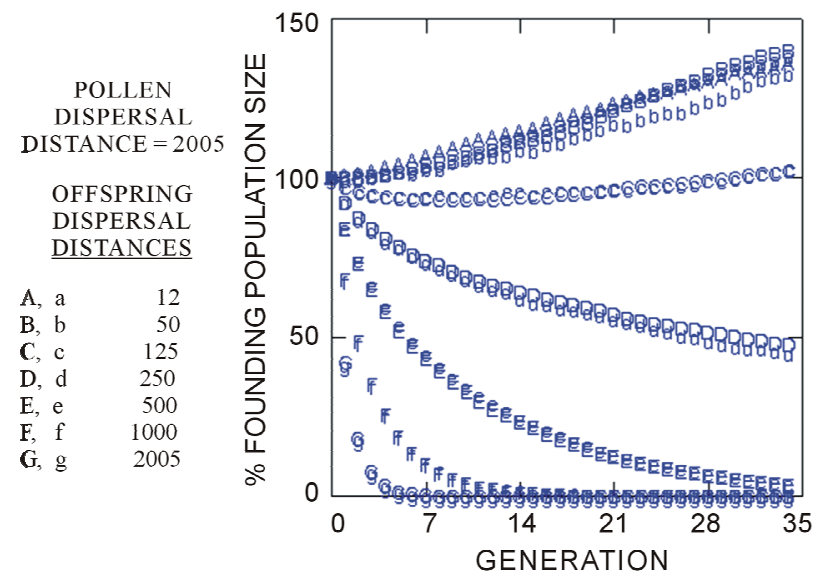

Figure 4. Continuing population size as a percent of the original number of founders remaining through generations for NEWGARDEN populations initiated with 1000 founders (letters in upper case) or $\mathbf{3 0 0}$ founders (lower case) scattered at random throughout the preserve. In these trials, maximum pollen dispersal is always 2005 grid points (greater than the length of one side of the preserve), while maximum offspring dispersal distances (in grid points) vary as indicated in the key to the left of the graft. See text for more details. 


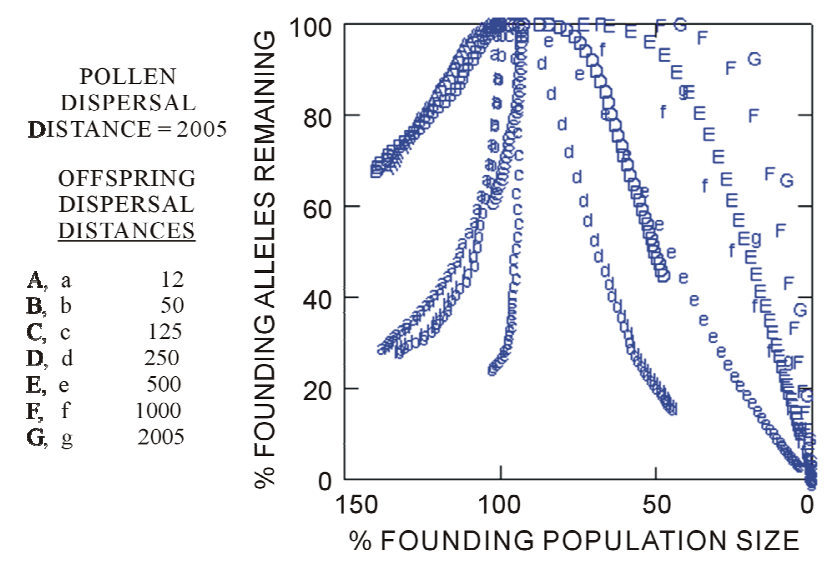

Figure 5. Percent of the founding alleles remaining relative to the size of the continuing population as a percentage of the original number of founders for the same trials for which population development data are depicted in Figure 4. These NEWGARDEN populations were initiated with 1000 founders (letters in upper case) or $\mathbf{3 0 0}$ founders (lower case) scattered at random throughout the preserve. In these trials, maximum pollen dispersal is always 2005 grid points (greater than the length of one side of the preserve), while maximum offspring dispersal distances (in grid points) vary as indicated in the key to the left of the graft. When the percent of founding alleles remaining has a value of $80 \%$, the percentage of stand members remaining relative to the founding population size ranges from approximately $128 \%$ (trial A) to $13 \%$ (trial G).

shown). Relatively long distance gene dispersal via pollen in all of these trials appears to prevent the higher $\mathrm{F}$ values seen when offspring and pollen dispersal were more limited (Figure 3).

\section{Discussion}

The NEWGARDEN trial examples presented here demonstrate that, all else equal, varying founder number and initial population density, as well as realized offspring and pollen dispersal distances, can have pronounced effects on population size change, retention of alleles, and degree of inbreeding and subdivision in isolated fragments through generations. In the processes of fragmentation or initiation of endangered species rescue populations, populations beginning with the same number of founders can develop along many different population size change and genetic diversity trajectories, depending on gene dispersal characteristics. Genetic diversity losses experienced by an isolated population will set the diversity potential for that population going forward. Populations differing in gene dispersal characteristics can be reduced to the same size but retain differing amounts of the founding genetic variation such that even if both populations expand to large equal numbers in the future, they will likely differ in the amount of genetic variation they hold. As shown in Figure 3, fragmented populations initiated with the same number of founders that then decline to the same size can have large differences in levels of inbreeding and differentiation due to gene dispersal differences, demonstrating the earlier noted principle that $\mathrm{N}_{\mathrm{c}}$ does not equal $\mathrm{N}_{\mathrm{e}}$. Judging the "conservation health" of a stand is best not estimated based on census number only.

Increased losses of genetic diversity promote evolution via drift, reduce the effectiveness of selection and close potential future avenues of evolution. While in the examples here we have used loci with alleles at low frequencies, we have found in other NEWGARDEN studies [11, and in prep.] that increased losses of low frequency alleles translate to greater drift-driven variance in allele frequencies for more common alleles under the same trial conditions, with such inflated variance also promoting the effects of drift over those of selection.

The trial examples presented here are meant to be heuristic, not exhaustive. Our goal is to demonstrate, through particular examples, that in fragmentation or restoration situations, especially where founders are limited, it is crucial to explore how realized gene dispersal distances can affect future outcomes for the population. The creation of fragments or rescue populations will often alter gene dispersal distances from those commonly experienced by a species under consideration in more natural circumstances. The trials examined here emphasize that obtaining accurate data on gene dispersal for targeted endangered rescue species can improve modeling to determine best restoration practices. NEWGARDEN analyses can be used to examine how varying preserve size or shape, founder number and genetic diversity, life history characteristics such as age dependent mortality and reproduction, and gene dispersal distances, can interact to affect population size and genetic diversity change. As shown above, previous trends in allele loss for currently similar size populations are not always obvious. NEWGARDEN can thus also be used to attempt to model the suspected conditions under which a population has developed to estimate current levels of genetic diversity. Such analyses might suggest beneficial population manipulations such as supplementing the population with more individuals, or with individuals from different genetic backgrounds, or altering local densities, promoting or limiting offspring dispersal, or altering pollen flow (e.g., hand pollinations or altering local pollination vector conditions). While we understand that a multitude of deterministic and stochastic factors beyond those modeled by NEWGARDEN can also impact the development of populations rendering precisely accurate simulations more difficult, we hope that NEWGARDEN analyses can contribute to developing first estimates of best prac- 
tices in planning endangered species protection and restoration projects. The NEWGARDEN program and associated materials are available for free at http://math.uc.edu/ pelikan/NEWGARDEN.

\section{Acknowledgements}

Both authors contributed equally to this work. We thank A. Buck, C. Daley, S. Heywood, Y. Kashimshetty, M. Simkins, and the Department of Mathematical Sciences and the Department of Biological Sciences at the University of Cincinnati. We thank the Charles Phelps Taft Research Center and the Ohio Plant Biotechnology Consortium for partial funding.

\section{REFERENCES}

[1] R. Frankham, J. D. Ballou and D. A. Briscoe, "Introduction to Conservation Genetics," 2nd Edition, Cambridge University Press, Cambridge, 2010. http://dx.doi.org/10.1017/CBO9780511809002

[2] F. W. Allendorf, G. Luikart and S. N. Aitken, "Conservation and the Genetics of Populations," 2nd Edition, Wiley-Blackwell, Hoboken, 2012.

[3] I. R. Franklin, "Evolutionary Changes in Small Populations," In: M. E. Soulé and B. M. Wilcox, Eds., Conservation Biology: An Evolutionary-Ecological Perspective, Sinauer, Sunderland, 1980, pp. 135-149.

[4] M. E. Soulé, "Thresholds for Survival: Maintaining Fitness and Evolutionary Potential," In: M. E. Soulé and B. M. Wilcox, Eds., Conservation Biology: An Evolutionary-Ecological Perspective, Sinauer, Sunderland, 1980, pp. 151-170.

[5] R. Frankham, "Effective Population Size/Adult Population Size Ratios in Wildlife: A Review," Genetical Research, Vol. 66, No. 1, 1995, pp. 97-107.

[6] R. S. Waples, "Definition and Estimation of Effective Population Size in the Conservation of Endangered Species," In: S. R. Beissinger and D. R. McCullough, Eds., Population Viability Analysis, University of Chicago Press, Chicago, 2002, pp. 147-168.

[7] D. Hedgecock, "Does Variance in Reproductive Success Limit Effective Population Sizes of Marine Organisms?" In: A. R. Beaumont, Ed., Genetics and Evolution of Aquatic Organisms, Chapman and Hall, London, 1994, pp. 122-134.

[8] R. Lande, "Mutation and Conservation," Conservation Biology, Vol. 9, No. 4, 1995, pp. 782-791.

[9] M. Lynch, J. Conery and R. Bürger, "Mutation Accumulation and the Extinction of Small Populations," The American Naturalist, Vol. 146, No. 4, 1995, pp. 489-518.

[10] Y. Willi, "Mutational Meltdown in Selfing Arabidopsis lyrata," Evolution, Vol. 67, No. 3, 2012, pp. 806-815.

[11] S. H. Rogstad and S. Pelikan, "Genetic Diversity in Establishing Plant Populations: Founder Number and Geometry," Science Publishers, Enfield, 2011.

[12] S. Pelikan and S. H. Rogstad, "NEWGARDEN: A Computer Program to Model the Population Dynamics and Genetics of Establishing and Fragmented Plant Populations," Conservation Genetics Resources, Vol. 5, No. 1, 2013, pp. 857-862. http://dx.doi.org/10.1007/s12686-013-9869-9

[13] D. L. Hartl, "A Primer of Population Genetics," 2nd Edition, Sinauer Associates, Inc., Sunderland, 1987. 